\title{
复杂非均质体参数化模型的切片及支撑点求解
}

\author{
陈 龙 ${ }^{1}$ 韩文瑜 $^{1}$ 何文涁 ${ }^{1}$ 徐 岗 ${ }^{2}$ \\ (1. 上海理工大学机械工程学院 上海 200093; \\ 2. 杭州电子科技大学计算机学院 杭州 310018)
}

\begin{abstract}
摘要: 针对复杂非均质 NURBS 体参数化模型(以下简称 HNURBS 体)的三维打印, 给出切片及支撑点求解算法。首先构建复 杂 HNURBS 体, 实现材料信息连续可视化表达。对 HNURBS 体进行六面体单元采样, 并和一系列切平面进行求交运算, 通 过截面边界轮廓的提取, 得到单连通和多连通截面区域轮廓及内部材料信息的参数化表达。对相邻的两切片轮廓进行布尔运 算, 得到三维打印模型的支撑点添加位置。实例结果表明, 算法能准确高效地生成复杂 HNURBS 体的切片数据及支撑点位 置, 从而为多材料实体三维打印提供技术支持。
\end{abstract}

关键词: 三维打印; 复杂非均质模型; 切片; 支撑点

中图分类号: TH164; TP391

\section{The Algorithm of Slicing and Obtaining Support Points for Complex Heterogeneous Volume Parameterization Model}

\author{
CHEN Long $^{1}$ HAN Wenyu ${ }^{1}$ HE Wenbin ${ }^{1}$ XU Gang ${ }^{2}$
}

(1. School of Mechanical Engineering, University of Shanghai for Science and Technology, Shanghai 200093;

2. School of Computer Science and Technology, Hangzhou Dianzi University, Hangzhou 310018)

\begin{abstract}
In order to make three dimensional printing for complex heterogeneous NURBS volume parameterization model (HNURBS volume) possible, a slicing and support points obtaining algorithm is proposed. First, a complex HNURBS volume is constructed, and the continuous and visual expression of material information is realized. The HNURBS volume is sampled by hexahedron element mesh method and intersected with a set of tangent planes. Then the parametric expression of the contours of simply-connected and multi-connected sections and internal material information is obtained by extracting slicing contours. Last, Boolean operations on two adjacent slicing contours are carried out to obtain the location of support points for three dimensional printing model. The example shows that the algorithm can accurately and efficiently generate slicing data and support points of the complex HNURBS volume, which provides technical support for three dimensional printing of the multi-material solid model.
\end{abstract}

Key words: three dimensional printing; complex heterogeneous volume; slicing; support points

\section{0 前言}

增材制造技术或三维打印技术诞生于 20 世纪 80 年代后期, 采用材料堆积的方法来制造产品, 因 为其在复杂结构制造和快速成型方面的突出表现, 被称为 20 世纪制造业领域的一项重大成果 ${ }^{[1-2]}$, 目 前在生物医学、航空航天、建筑、科技文化等领域 得到快速发展及广泛应用 ${ }^{[3-6]}$ 。目前三维打印产品主 要有两种, 一是由单一材料构成的均质产品, 二是

* 国家自然科学基金(51475309，61772163)和浙江省杰出青年自然科学基 金(LR16F020003)资助项目。20181111 收到初稿, 20190409 收到修改稿
由多种材料构成的非均质产品，包括成分和结构呈 连续梯度变化的梯度功能材料。梯度功能材料因为 梯度功能的涂覆型、连接型、整体型而在各个领域 具有十分广阔的应用前景 ${ }^{[7]}$ 。目前三维打印技术能 够处理的模型主要是以 STL 数据格式保存的 B-Rep 模型, 但是 STL 数据结构只包含点与面的信息, 因 此在添加多材料组分方面存在较大困难。

以 NURBS 作为基函数的体参数化模型在实体 表达方法因具有较大的优势, 近年来受到较多重视。 体参数化模型的控制点控制模型的几何形状，为控 制点添加材料特征, 通过 NURBS 基函数实现材料 和几何的并行设计, 构成材料域映射到物理域, 实 现材料的连续性表达, 完成 HNURBS 体的模型构 
建。NURBS 高阶连续性保证了存在许多有效且数 值稳定的算法来产生和优化体参数模型, 而且基于 该模型的等几何分析方法具有强大的灵活性、优异 的精确性和收玫性等优势, 因此一经提出在各领域 得到应用及推进 ${ }^{[8]}$ 。

针对 HNURBS 体的三维打印, 切片处理是其中 关键步骤。随着产品材料组分和几何结构愈加复杂, 在打印产品模型的悬空部位时，材料因悬在空中而 无法融合, 因此需要在切片前对物体模型悬空部位 下方添加支撑结构，作为物体模型的一部分被打印 机打印。尽管目前已有不少体参数化模型构建的方 法, 但是复杂 HNURBS 体的构建则较为困难, 因此 现有切片算法也多为面向 B-Rep 模型, 针对复杂 HNURBS 体的研究则很少。

本文针对复杂 HNURBS 体, 提出模型三维打印 过程中的切片及支撑点求解算法。如图 1 所示, 首 先根据 NURBS 体参数化模型的定义, 利用多片体 参数化模型构建方法, 完成 HNURBS 体构建以及材 料连续性可视化表达。对体参数化模型进行六面体 单元划分后进行切片操作和材料信息插值计算, 得 到切片截面信息。通过对截面信息拓扑信息重构, 完成截面边界轮廓的提取及拟合。根据切片相邻两 截面的布尔运算结果, 完成模型的支撑求解。过程 如图 1 所示。

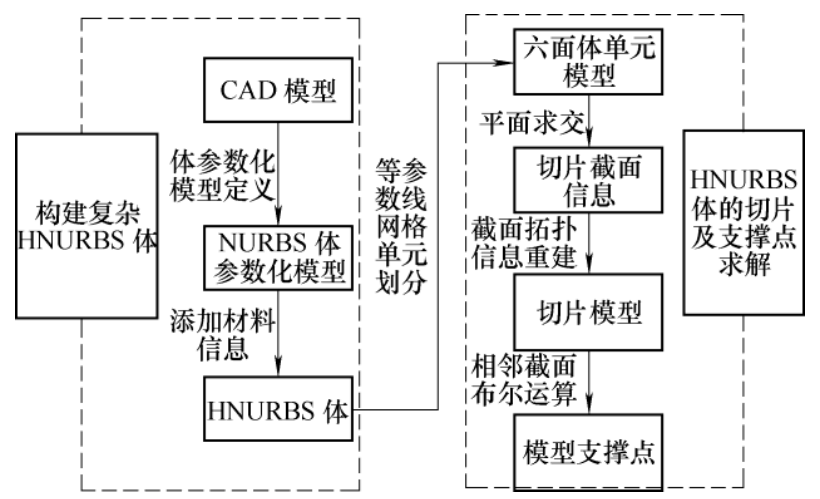

图 1 非均质产品的三维打印技术

\section{1 相关工作}

\section{1 非均质体造型}

非均质产品要求实现结构与功能一体化的优化 设计。结构包括拓扑和几何,功能则由结构和材料成 分体现, 因此非均质产品是一种典型的要求实现几 何、拓扑、材料一体化设计的产品。从几何表达角 度出发, 已提出多种设计方法, 主要有: B-Rep 模 型或者 CSG 模型 ${ }^{[9]}$ 、基于 CATIA 等现有 CAD 软件 提供的模型 ${ }^{[10]}$ 、精确边界模型 ${ }^{[11]}$ 。诸多几何设计方
法大多围绕具有平面或二次曲面等规则外表面的传 统零件展开, 较少涉及外形或者拓扑复杂的产品。 从材料表达角度出发, 也已提出多种设计方法: 材 料源法 ${ }^{[12]}$ 、样条法 ${ }^{[13]}$ 、优化法 ${ }^{[14]}$ 。诸多材料设计方 法缺少直观便捷的材料设计工具，同时微观结构分 布与宏观性能缺乏直接关联, 使得设计得到的产品 几何和材料分布都非常理想化, 产品的实用性也受 到一定限制。

\section{2 体参数化建模}

COHEN 等 ${ }^{[15]}$ 提出了分析意识建模的概念, 该建模方法中, CAD 模型的参数化表达必须能够 适用于等几何分析。MARTIN 等 ${ }^{[16]}$ 提出了利用体 积调和函数将三角网格模型拟合成 B 样条体的方 法。AIGNER 等 ${ }^{[17]}$ 提出了一种构造扫掠体 NURBS 参数化的变分方法。WANG 等 ${ }^{[18]}$ 将分治、约束聚 合和分层优化技术相结合，从六个边界 $\mathrm{B}$ 样条曲 面上得到有效的三变量 $\mathrm{B}$ 样条实体。XU 等 ${ }^{\left[{ }^{[19}\right.}$ 提 出了用均匀性改进的边界重新参数化的方法构建 适于分析的体参数化模型的两阶段方案。最近, 在给定模板域的情况下，针对抽象特征相似的一 组模型, 提出了一种基于 $\mathrm{B}$ 样条的一致体参数化 方法 ${ }^{[20-21]}$ 。

\section{3 三维打印切片及支撑求解}

切片算法一个基础问题是如何针对具有不同输 入格式的模型进行可改变层厚的切片 ${ }^{[22]}$ 。现有切片 算法大多针对以 STL 数据格式保存的 B-Rep 模 型 ${ }^{[23-24]}$ 。文献[25]介绍了一种针对 F-Rep 模型的 直接切片算法, 避免了对三角形网格的存储, 在 一定程度上较 B-Rep 模型更加容易作为几何数据 文件的输入。文献[27]则提出针对 NURBS 曲面的 自适应切片算法, 且在精准且光顺的表面模型进 行了验证。

切片的支撑求解是另外一个重要基础问题, 目 前一般有两种方法实现支撑求解。第一种为现有许 多切片引擎如 Cura、MeshMixer 等采用的方法 ${ }^{[27-28]}$ 。 该方法选取三维物体模型中所有外法向朝下且与水 平面夹角较小的面(即接近水平的面)作为需要添加 支撑的部位 ${ }^{[29-30]}$ 。另一种方法是对两个连续切片层 作布尔运算, 结果通常会得到一个点集表示需要支 撑的点, 通过计算切片分组、边界框计算、补偿和 布尔运算，得到支撑点位置 ${ }^{[31-32]}$ 。

体参数化模型因其在连续非均质材料表达方面 具有独特的优良性质而逐渐受到重视。但因受制于 复杂体参数化模型构建的困难，使得面向复杂的非 均质体参数化模型的切片算法及支撑求解方面并没 得到较好研究。 


\section{2 复杂 HNURBS 体表达与构建}

\section{1 单片 HNURBS 体定义}

NURBS 体参数化模型是通过创建一个三变量 张量体参数化模型 $T$ 和一个规则的封闭体(立方 体) $P$ 建立一一映射关系。如图 2 所示, $T$ 指具有物 理域边界, 建立在 $(X, Y, Z)$ 坐标系统中的三维模型, 规整的封闭体 $P$ 指的是由正交参数化系统 $(U, V, W)$ 构建的规整的立方体，(1)为物理域到参数域的映射 关系, (2)为参数域到物理域的映射关系, (3)为物理 域一单元在参数域对应的单元。其映射函数即三维 空间 NURBS 体参数化模型的表达式如下。

$$
\begin{gathered}
B(u, v, w)= \\
\frac{\sum_{i=0}^{n} \sum_{j=0}^{m} \sum_{k=0}^{l} N_{i, p}(u) N_{j, q}(v) N_{k, r}(w) \omega_{i, j, k} P_{i, j, k}(x, y, z)}{\sum_{i=0}^{n} \sum_{j=0}^{m} \sum_{k=0}^{l} N_{i, p}(u) N_{j, q}(v) N_{k, r}(w) \omega_{i, j, k}}
\end{gathered}
$$

这里 $\left\{P_{i, j, k}\right\}$ 为体控制点, $\left\{\omega_{i, j, k}\right\}$ 是权因子, $N_{i, p}(u), N_{j, q}(v), N_{k, r}(w)$ 分别是定义在非周期(且非 均匀)节点矢量空间 $U, V, W$ 上的次数分别为 $p, q, r$ 次的 NURBS 基函数。

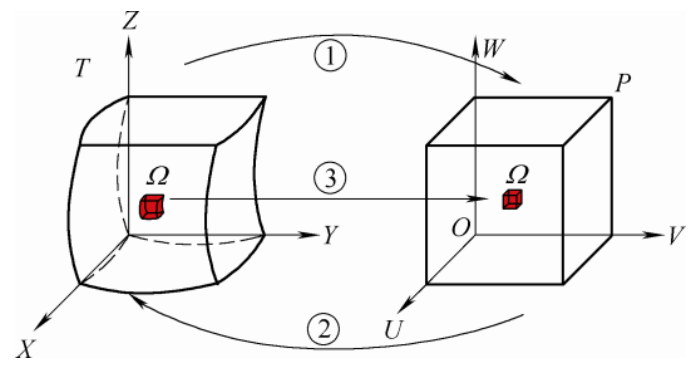

图 2 参数域与物理域的映射关系

$U, V, W$ 的节点矢量空间

$U=\left[u_{0}=u_{1} \cdots=u_{p}, u_{p+1}, \cdots, u_{n}, u_{n+1}=u_{n+2} \cdots=u_{n+p+1}\right]$

$V=\left[v_{0}=v_{1} \cdots=v_{q}, v_{q+1}, \cdots, v_{m}, v_{m+1}=v_{m+2} \cdots=v_{m+q+1}\right]$

$W=\left[w_{0}=w_{1} \cdots=w_{r}, w_{p+1}, \cdots, w_{l}, w_{l+1}=w_{l+2} \cdots=w_{l+r+1}\right]$

下式为 $U$ 方向上的 NURBS 基函数的表达式, $V, W$ 方向上的基函数定义与此类似。

$\left\{\begin{array}{l}N_{i, p}(u)= \begin{cases}1 & u_{i} \leqslant u \leqslant u_{i+1} \\ 0 \quad & \text { 其他 }\end{cases} \\ N_{i, p}(u)=\frac{u-u_{i}}{u_{i+p}-u_{i}} N_{i, p-1}(u)+\frac{u_{i+p+1}-u}{u_{i+p+1}-u_{i+1}} N_{i+1, p-1}(u)\end{array}\right.$

HNURBS 体相比较于 NURBS 体参数模型, 除 了表达实体的几何信息, 还需表达实体的材料信息。 假定考虑两组份材料形成的材料混合形成 HNURBS
体, 将组分百分比含量作为变量赋予控制点, 则任 意位置材料成分可以由以下公式确定。

$$
B(u, v, w, h)=
$$

$$
\frac{\sum_{i=0}^{n} \sum_{j=0}^{m} \sum_{k=0}^{l} N_{i, p}(u) N_{j, q}(v) N_{k, r}(w) \omega_{i, j, k} P_{i, j, k}(x, y, z, \lambda)}{\sum_{i=0}^{n} \sum_{j=0}^{m} \sum_{k=0}^{l} N_{i, p}(u) N_{j, q}(v) N_{k, r}(w) \omega_{i, j, k}}
$$

式中, $h$ 和 $\lambda$ 分别表示实体点 $B(u, v, w)$ 和控制点 $P_{i, j, k}(x, y, z)$ 上的其中一种材料组分信息, 另外一 种材料信息为 $1-h$ 和 $1-\lambda$ 。对于三组分材料信息, 只需加一个参数表示材料信息即可。采用图像处 理中的灰度和三原色概念, 将两组分 HNURBS 体 的材料成分转化为灰度, 将三组分材料成分转化 为红色 $(R)$ 、绿色 $(G)$ 、蓝色 $(B)$ 三种颜色, 从而实 现 HNURBS 体的材料信息连续可视化表达。因此 如何求取控制点材料灰度信息是 HNURBS 体构 建的重点。

\section{2 复杂 HNURBS 体的构建}

对于几何, 材料结构复杂的体参数化模型, 单 片 NURBS 体参数化模型表达比较困难, 一般需将 模型分割为多片六面体块，每片六面体块 $T$ 都与一 个规则的参数域 $P$ 形成一一映射的关系。文献 [32] 中提供了一套从三角形网格模型构建复杂体参数化 模型的通用方法。通过最小化三角形网格模型的 $\ell_{1}$ 范数, 利用抽象结构得到简化网格模型的多立方体 结构。采用样条拟合技术构造细分曲面, B 样条体 参数化模型填充立方体结构, 从而得到 $C^{1}$ 连续的多 片体参数模型，具有良好的鲁棒性。图 3 所示为由 $T_{1} 、 T_{2} 、 T_{3}$ 单片 NURBS 体参数化拼接构建的 $\mathrm{L}$ 形 体参数化模型 $T$, 每片 NURBS 体参数化模型与参 数域 $P$ 形成一一映射的关系。

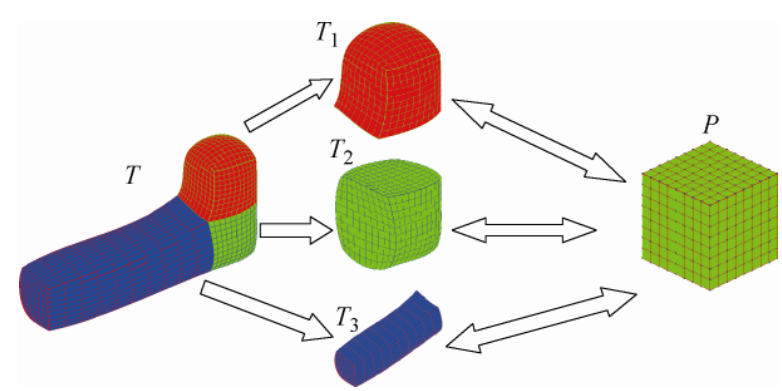

图 3 多片 NURBS 体的构建与映射关系

文献[33]中对模型进行 CT 扫描后切片采样, 得 到几何、材料信息离散的截面点，根据曲面拟合技 术对切片截面进行材料拟合。控制点材料信息的求 取主要分为两方面: 利用包围盒近似方法对采样点 
的几何空间进行耦合, 即利用采样点的几何信息反 求参数值; 根据最小二乘法原理利用采样点的参数 值以及材料信息反求控制点的材料信息。构建截面 与控制点材料信息的约束优化方程

$$
\Gamma\left(\lambda_{i j k}\right)=
$$

$$
\begin{gathered}
\min \sum_{s=1}^{N}\left(B(h)-\sum_{i=0}^{n} \sum_{j=0}^{m} \sum_{k=0}^{l} N_{i, p}(u) N_{j, q}(v) N_{k, r}(w) P_{i, j, k}(\lambda)\right)^{2} \\
\text { s.t. } \quad 0 \leqslant \lambda_{i j k} \leqslant 1
\end{gathered}
$$

利用上述方法实现 HNURBS 体的构建, 图 4 所示为两组份材料的 HNURBS 体可视化实例, 图 $4 \mathrm{a}$ 为含有材料信息的控制点灰度图, 图 $4 \mathrm{~b}$ 为构建 的 HNURBS 体, 图 4c 为分割的 HNURBS 体内部灰 度图。

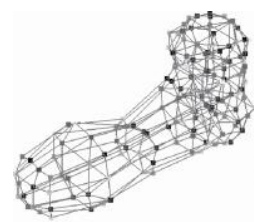

(a)

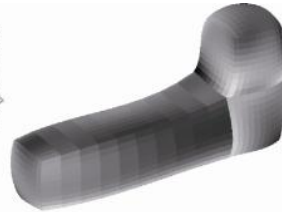

(b)

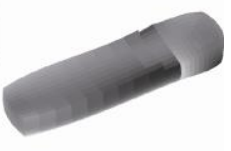

(c)
图 $4 \mathrm{~L}$ 模型的 HNURBS 体

\section{3 复杂 HNURBS 体切片算法}

切片是通过一系列间隔一定距离的平面与 HNURBS 体求交的过程。如图 5 所示, 首先将 HNURBS 体划分为六面体单元模型, 求取六面体单 元与切平面的交点形成截面, 然后对截面进行拓扑 重建和边界曲线拟合, 得到 HNURBS 体在该平面上 的截面轮廓。

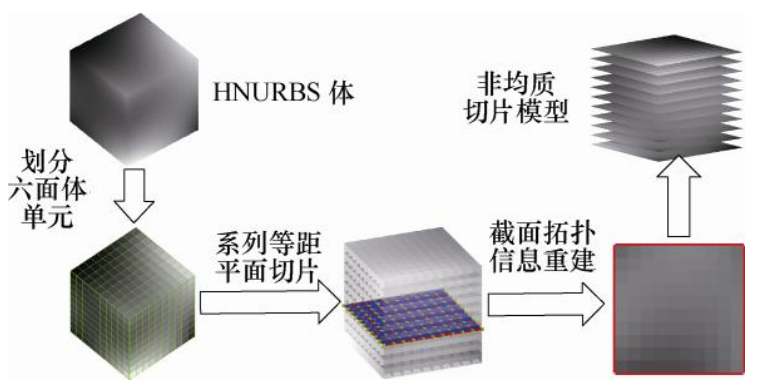

图 5 切片原理图

\section{1 六面体单元模型生成}

单片 NURBS 体参数化模型物理域 $T$ 与参数域 $P$ 具有一一映射的关系, 沿参数域 $u, v, w$ 三个参数 方向, 将节点矢量空间 $U, V, W$ 细分为 $\delta_{u}, \delta_{v}, \delta_{w}$ 等 份, 获得等参数线网格单元, 对应于物理域则沿三 变量张量方向细分获得六面体单元, 如图 6 所示。

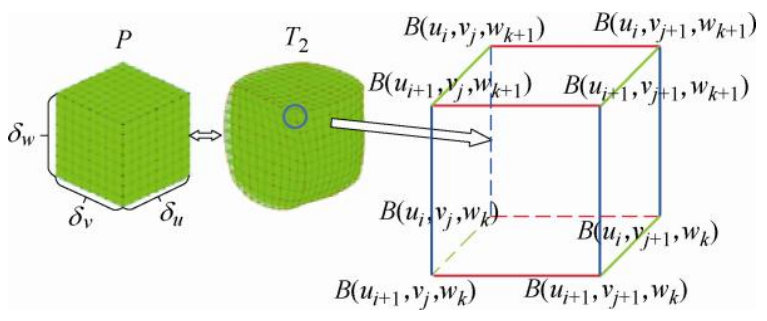

图 6 六面体单元划分

对于多片体参数化模型构成的复杂 HNURBS 体，在满足几何、材料精度的情况下，对每片 HNURBS 体进行六面体单元划分。如图 6 所示，当 $\delta_{u}, \delta_{v}, \delta_{w}$ 细分次数足够大, 则可认为由 8 个等参点 $B\left(u_{i}, v_{j}, w_{k}\right) ， B\left(u_{i+1}, v_{j}, w_{k}\right), B\left(u_{i}, v_{j+1}, w_{k}\right)$ ， $B\left(u_{i+1}, v_{j+1}, w_{k}\right) \quad B\left(u_{i}, v_{j}, w_{k+1}\right), B\left(u_{i}, v_{j+1}, w_{k+1}\right)$, $B\left(u_{i}, v_{j+1}, w_{k+1}\right), B\left(u_{i+1}, v_{j+1}, w_{k+1}\right)$ 构建的六面体单元 更接近于规则六面体。

\section{2 求取单元原始截面点}

为求取截面数据点, 需求切平面与所有相交六面 体单元的相交点。将六面体单元边按三个参数方向分 为红绿蓝三组, 每组有 4 条连线, 计算单元每条连线 与平面的交点，如图 7 所示。已知线段两端点的材料 成分，利用线性插值方法即可得到交点处的材料信 息。在非退化情况下最终得到的切平面与一个六面体 单元相交的情况如图 8 所示, 截面形状分为以下四 类: 三角形(图 8a), 四边形(图 8b), 五边形(图 8c), 六边形(图 8d), 均为凸多边形。在退化情况下, 平面 与六面体单元相交于一个顶点时, 顶点作为原始截面 点, 相交于一条棱边时, 棱边作为边界线段单独考虑, 相交于一个平面时，以该面作为单元截面。

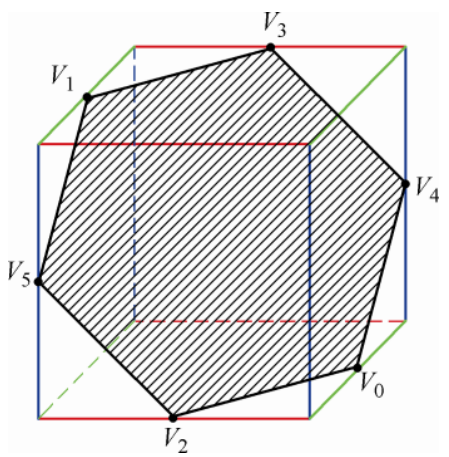

图 7 截面点几何、材料信息计算

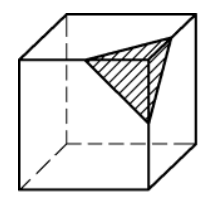

(a)

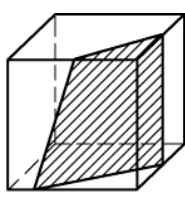

(b)

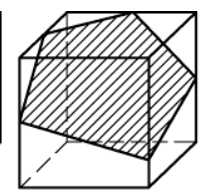

(c)

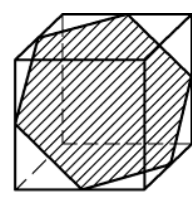

(d)
图 8 非退化情况下平面与六面体单元截面形状

如图 7 所示，切平面与某六面体单元相交，其 截面形状为一凸 $n(3 \leqslant n \leqslant 6)$ 边形，含有材料信息的 
截面点坐标分别为 $V_{i}(0 \leqslant i \leqslant 5)$ 。

\section{3 单元截面点排序}

经过上述求交过程得到的截面点是杂乱无序 的, 需要对其排序, 以便连接这些截面点形成封闭 边界。六面体单元的 8 个顶点按等参点采样得到, 不存在重复或重叠的顶点, 因此根据矢量夹角大小 完成凸多边形的截面点排序。如图 9 所示, 首先得 到凸多边形的几何中心 $P$, 以 $\boldsymbol{V}_{0} \boldsymbol{P}$ 矢量作为基准矢 量, 分别求解 $V_{1} P, V_{2} P, V_{3} P, V_{4} P, V_{5} P$ 与 $V_{0} P$ 矢量的夹角来确定排列顺序, 最后连接排序后的点 形成封闭边界。

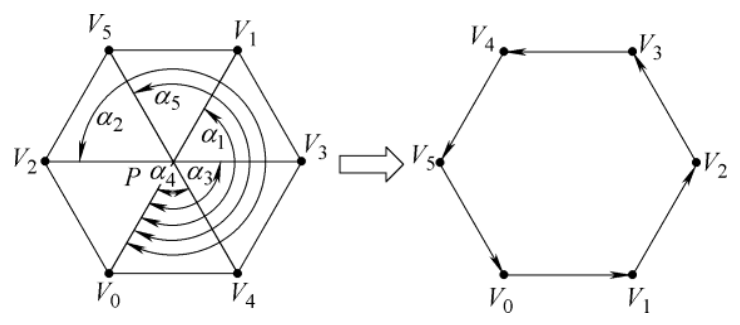

图 9 凸多边形顶点排序

同一个切平面会和多个六面体单元相交, 因此 需要将单个六面体单元得到的截面拼接在一起, 得 到具有封闭边界的截面。如图 10 所示, 共 $A, B, C, D, E, F, G, H$ 一共 8 个六面体单元, 每个单元对 应的截面 $A$-(1), $C$-(2), $D$-(3), E-(4),F-(5), $G$-(6),H-(7)。

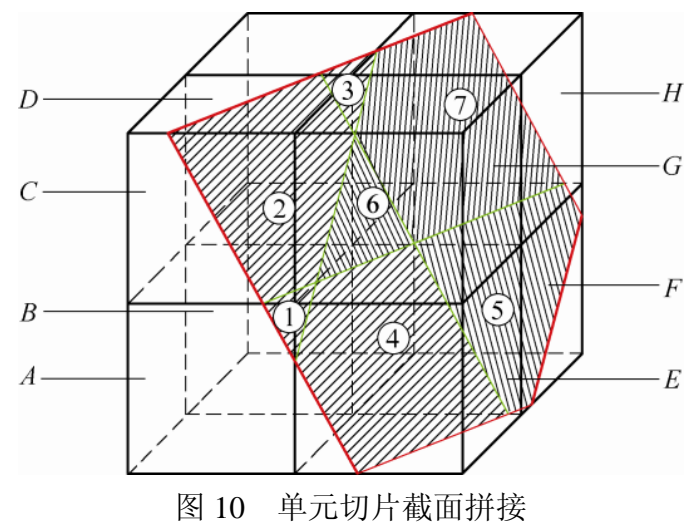

\section{4 封闭边界轮廓识别}

切片得到的截面数据有可能是单连通区域, 也 可能是多连通区域。首先需要查找所有封闭区域边 界轮廓信息, 然后判断边界轮廓之间的包含关系, 从而建立多连通区域。封闭区域边界轮廓查找分为 两个主要步骤: 一是边界轮廓线段查找, 二是边界 轮廓线段排序与连接。

(1) 边界轮廓线段查找。

首先根据 HNURBS 体定义, 得到参数值等于 0 或 1 对应的点即为物理域的边界点。对于某段封闭 边界的两端点都属于边界点时, 该线段属于边界轮 廓线段。如图 $11 \mathrm{a}$ 所示, 截面(2)边界线段为
$V_{0} V_{1}, V_{1} V_{2}, V_{2} V_{3}, V_{3} V_{4}, V_{4} V_{0}$, 其中边界点为 $V_{0}, V_{1}, V_{2}$, 故 截面(2)边界轮廓线段为 $V_{0} V_{1}, V_{1} V_{2}$ 。搜寻其他所有边 界轮廓线段经排序与连接后构成截面的边界轮廓, 红色线段即为截面边界轮廓。

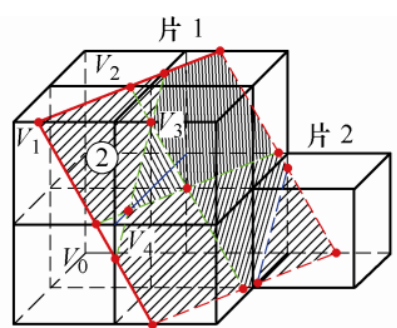

(a)

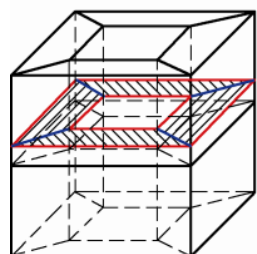

(b)

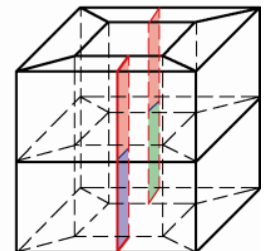

(c)
图 11 截面边界轮廓线段查找

尽管单个六面体单元的封闭边界线段是单片参 数化模型的边界轮廓线段，但未必是整个物理域的 边界轮廓线段, 出现线段数量 $\geqslant 2$ 且线段顶点被多 线段共享的 “伪边界轮廓线段”, 即图 12 中粗线段, 被相邻两个单元共享。尽管该类线段完全位于单片 图 11a 或者单个单元图 11b、11c 的 HNURBS 体边 界上, 但却不是整个模型的边界, 因此需要剔除此 类线段。图 $12 \mathrm{a} 、 12 \mathrm{~b}$ 分别为图 11b、11c 的伪边界 线段剔除过程，最终得到粗线段围成的边界轮廓。

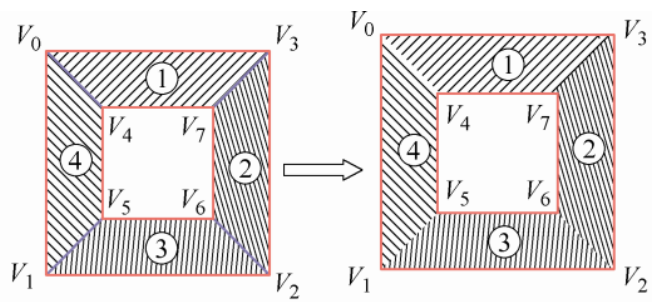

(a)
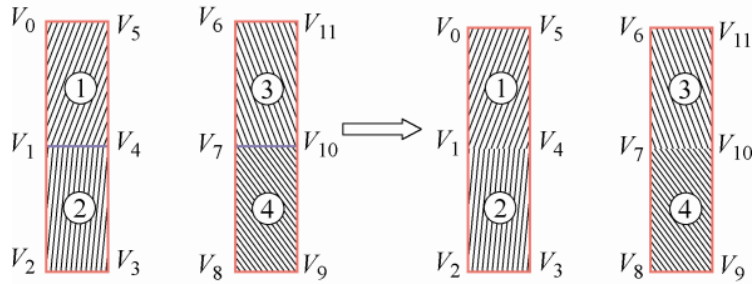

(b)

图 12 伪边界轮廓线段去除

(2) 边界轮廓线段排序与连接。

如图 12 所示去除伪边界轮廓线段后, 每一个边 界点被两条边界轮廓线段共享, 以边界点作为关键 
码, 以该边界点所在的两条线段的另两个顶点作为 值进行哈希表存储，如表 1、2 所示。

表 1 图 11a 边界轮廓哈希表

\begin{tabular}{ccc}
\hline 关键码 & 数值 & \\
\hline$V_{0}$ & $V_{1}$ & $V_{3}$ \\
$V_{1}$ & $V_{0}$ & $V_{2}$ \\
$V_{2}$ & $V_{1}$ & $V_{3}$ \\
$V_{3}$ & $V_{2}$ & $V_{0}$ \\
$V_{4}$ & $V_{7}$ & $V_{5}$ \\
$V_{5}$ & $V_{4}$ & $V_{6}$ \\
$V_{6}$ & $V_{5}$ & $V_{7}$ \\
$V_{7}$ & $V_{4}$ & $V_{6}$ \\
\hline
\end{tabular}

表 2 图 11b 边界轮廓哈希表

\begin{tabular}{ccc}
\hline 关键码 & 数值 & \\
\hline$V_{0}$ & $V_{1}$ & $V_{5}$ \\
$V_{1}$ & $V_{0}$ & $V_{2}$ \\
$V_{2}$ & $V_{1}$ & $V_{3}$ \\
$V_{3}$ & $V_{2}$ & $V_{4}$ \\
$V_{4}$ & $V_{3}$ & $V_{5}$ \\
$V_{5}$ & $V_{0}$ & $V_{4}$ \\
$V_{6}$ & $V_{7}$ & $V_{11}$ \\
$V_{7}$ & $V_{6}$ & $V_{8}$ \\
$V_{8}$ & $V_{7}$ & $V_{9}$ \\
$V_{9}$ & $V_{8}$ & $V_{10}$ \\
$V_{10}$ & $V_{9}$ & $V_{11}$ \\
$V_{11}$ & $V_{6}$ & $V_{10}$ \\
\hline
\end{tabular}

根据关键码查找相应的值, 再以值作为关键码 查找新的值, 以此顺序构建循环队列作为判定边界 点的顺序方向, 完成图 $11 \mathrm{~b}$ 边界轮廓的顺序构建 $V_{0} V_{1} V_{2} V_{3} V_{0}, V_{4} V_{5} V_{6} V_{7} V_{4}$, 图 11c 边界轮廓的顺序构建 $V_{0} V_{1} V_{2} V_{3} V_{4} V_{5} V_{0}, V_{6} V_{7} V_{8} V_{9} V_{10} V_{11} V_{6}$ 。如图 13 所示为图 $11 \mathrm{~b}$ 多段线边界轮廓依据哈希表构建的循环队列。

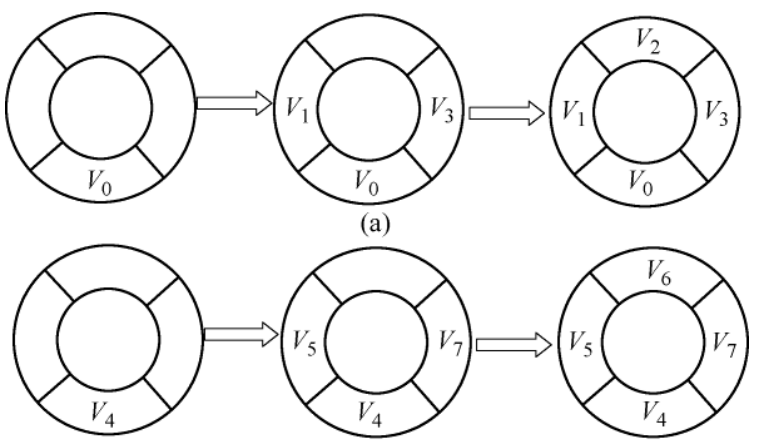

(b)

图 13 哈希表构建的循环队列

当 HNURBS 体片与片之间为线接触时, 如图 14 所示, 虽然切片区域轮廓中存在一个公共顶点被
多段线段共享，但仍将该区域分为两个独立区域。

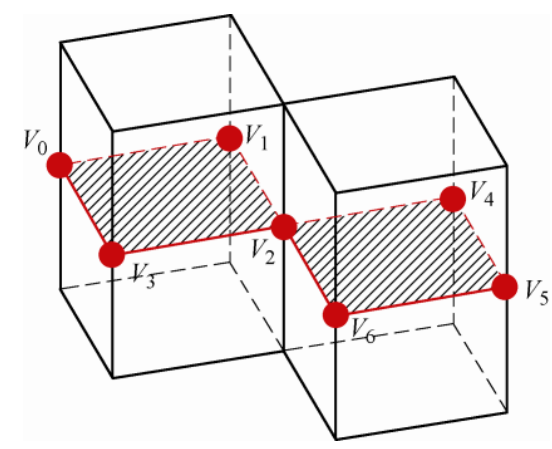

图 14 单连通区域退化情况

\section{5 多连通截面区域轮廓识别}

通过上述封闭区域边界轮廓线段查找与排序, 最终形成多个封闭环形边界轮廓区域, 如图 15a 所 示。对环进行编号 $L_{i}(i=0,1, \cdots)$, 每个环对应一个 封闭区域 $\Omega(i=0,1, \cdots)$, 如图 15c 所示。依据环上 的点是否在另一环的区域内, 判断环之间的包含关 系，构建多连通区域。以每个环作为一个节点, 构 建拓扑关系树，如图 15b 所示，一对父子节点表明 父节点包含子节点，兄弟关系的节点则表明两个节 点被同一父节点所包含。

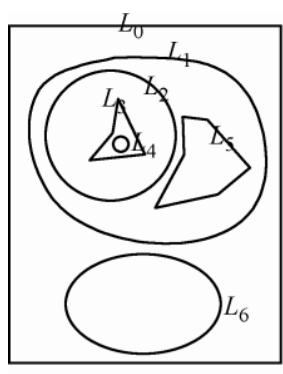

(a)

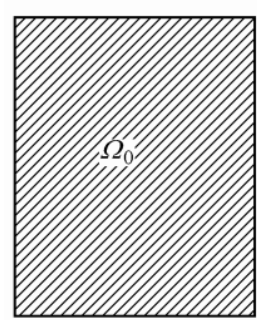

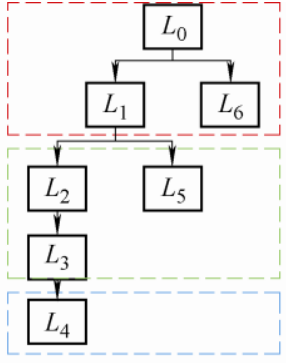

(b)

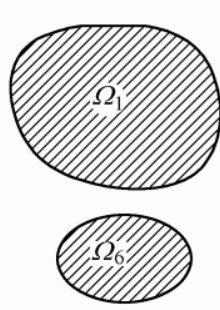

(c)

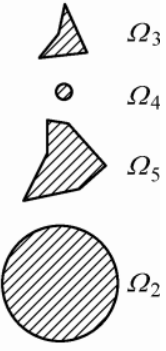

图 15 连通域编号及包含关系查找

将图 $15 \mathrm{~b}$ 中所有节点, 按照奇偶层次关系分组 来定义多连通区域的内外边界轮廓, 对子树内环对 应的拓扑区域进行布尔运算，主要包括父子节点之 间的差运算，满树时兄弟节点之间的交运算，非满 树时兄弟节点之间的并集运算，以及子树树根之间 的并集运算。如图 16 所示，依据环之间的拓扑关系 来确定连通区域, 图 16a 为第一棵满子树父子差运 算后进行兄弟交运算的过程, 可以看出 $L_{0}$ 是 $L_{1}$ 和 $L_{6}$ 内边界轮廓对应的外边界轮廓; 图 $16 \mathrm{~b}$ 为第二棵 
非满子树, 父子差运算后进行兄弟并运算的过程, $L_{3}$ 是外边界轮廓 $L_{2}$ 对应的内边界轮廓, $L_{5}$ 是外边界 轮廓。第三棵子树为本身的根节点, $L_{4}$ 是外边界轮 廓; 图 16c 为每棵子树进行并集运算后求解截面区 域的过程。

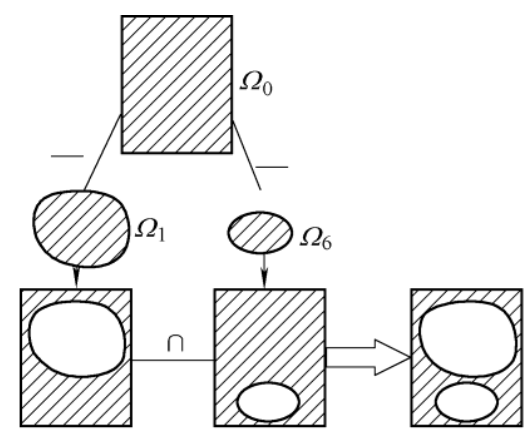

(a)
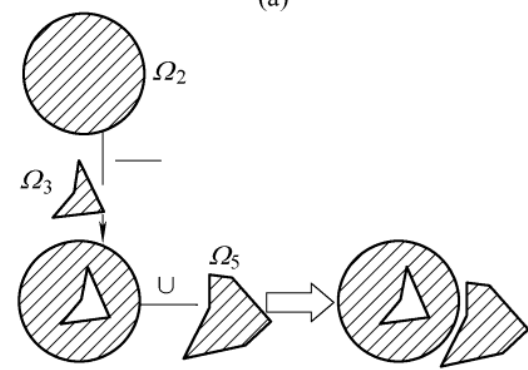

(b)

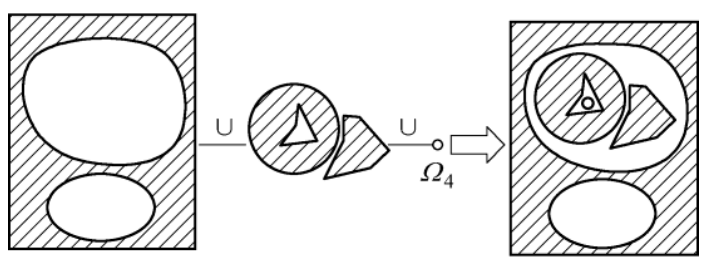

(c)

图 16 截面连通区域的布尔运算

\section{4 复杂 HNURBS 体支撑点求解}

\section{1 支撑点求解原理}

求解支撑点的原理如下: 将相邻两层截面投影 在垂直于生长方向上的某投影面上, 上一层截面某 区域如果落在下一层切片的轮廓之外, 则对应的两 层切片之间的区域需要添加支撑点。如图 17 所示为 相邻三层切片, 层厚为 $\delta$ 的切片, 第 $i-1$ 层为上一 层切片, 第 $i+1$ 层为下一层切片。求解第 $i$ 层的支

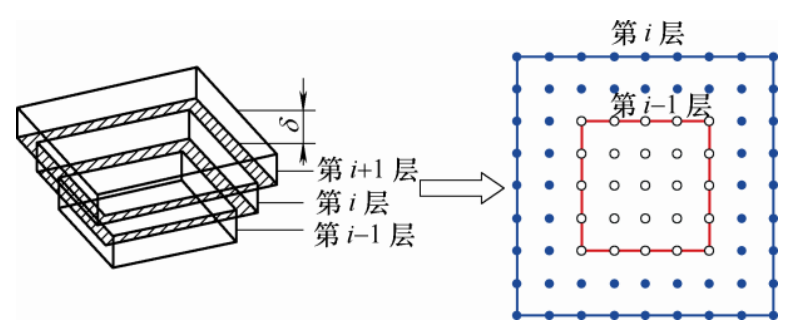

图 17 支撑点求解
撑点, 将三维截面转换到二维截面上, 判断第 $i$ 层 的截面点是否在第 $i-1$ 层的二维截面轮廓, 如果在 轮廓内, 证明该点在第 $i-1$ 层中存在材料支撑如 17 图中空心圆点, 如果不在, 则说明该点在上一层中 没有材料支撑，则为支撑点，如图 17 中实心圆点。

\section{2 支撑区域与支撑点求取}

支撑区域的求取是利用上下两层截面边界轮廓 求差实现的, 支撑点的求取是通过第 $i$ 层的截面点 与第 $i-1$ 层截面边界轮廓的位置关系判定。如图 18 所示为判断第 $i$ 层截面点 $A, B, C, D, E, F$ 与第 $i-1$ 层 轮廓 $V_{0} V_{1} V_{2} V_{3} V_{4} V_{5} V_{0}$ 的位置关系, 分为在轮廓内部、 外部、边界上。对于非边界点 $A, B, D, F$, 从该点沿 水平方向作辅助射线, 判断辅助射线与轮廓的交点 个数, 如果交点个数为偶数, 则点在轮廓外部, 如 点 $A, F$; 否则点在轮廓内部, 如点 $B, D$; 对于边界 点 $C, E$, 在第 $i-1$ 层肯定存在材料支撑, 属于在轮 廓内部。

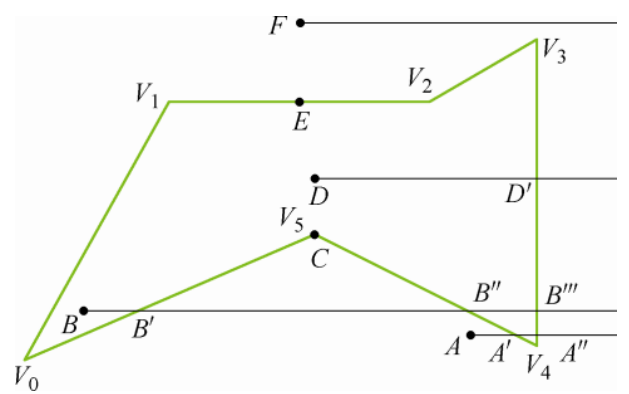

图 18 判断点是否在截面区域内

\section{5 设计实例}

本文以 VS2010+OpenGL 为编程平台, 开发了 一套非均质复杂参数化模型切片程序, 实现了复杂 HNURBS 体建模与切片功能, 并做出了若干实例。 如图 19 所示, 图 19a 表示在不同细分次数下的 HNURBS 体显示, 从左至右细分 5、10、20、25 次 的 L 模型, 随着细分次数的增加, HNURBS 体的几 何、材料精度明显增加。图 19b、19c、19d 分别为
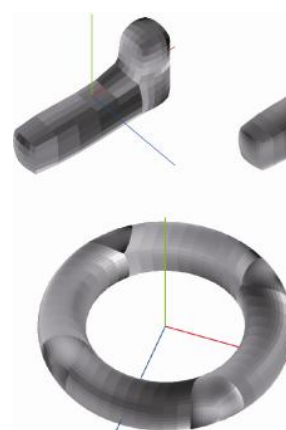

(b)

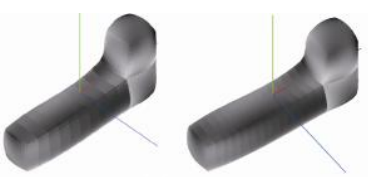

(a)
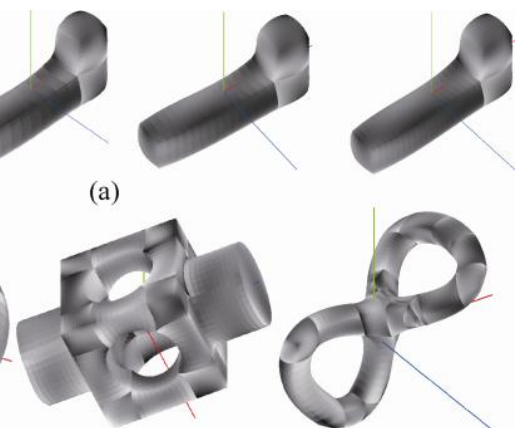

(c)

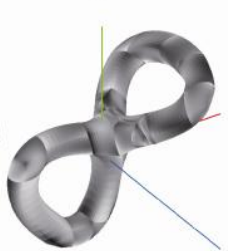

(d)
图 19 HNURBS 体的构建与显示 
分割为 8 片的 Torus 模型、24 片的 Block 模型、27 片的扭 Eight 模型, 模型以灰度图形式显示, 以表 现每个模型的材料成份。

在切平面与复杂 HNURBS 体相切过程中, 会得 到多连通域的切割截面。如图 20 所示为 Block 模型、 扭 Eight 模型在分图不同位置的截面和边界轮廓。 第一列中不同颜色表示每个位置的 $A 、 B 、 C$ 三个切 片，第 2、3、4 列分别表示三种颜色所示不同截面 的边界轮廓。

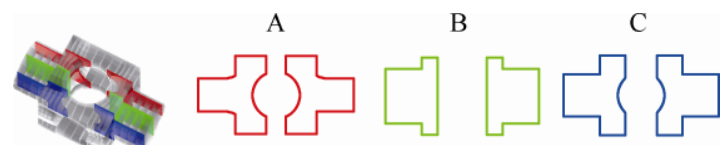

(a)

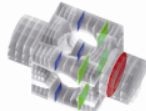<smiles>C1CCCCC1</smiles>

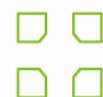

(b)
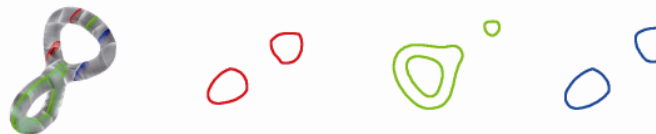

(c)
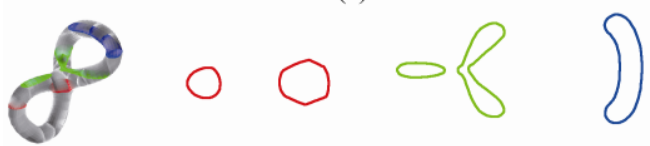

(d)

图 20 不同位置截面轮廓

表 3 所示为图 20c 扭 Eight 模型三个截面 A、B、 $\mathrm{C}$ 依次在细分 5 次、10 次、20 次、25 次的情况下, 切片截面单元数, 截面点数以及边界轮廓线段数量。 图 21 所示为 $\mathrm{B}$ 轮廓在不同细分次数下蓝色截面边 界轮廓线段和红色线段顶点。根据表 3 和图 21, 随 着细分次数的提高, 增加了与切片相交的截面单元 数, HNURBS 体切片截面的几何、材料精度, 边界 轮廓的几何精度相应提高。

表 3 不同细分次数的截面

\begin{tabular}{cccccc}
\hline \multirow{2}{*}{ 边界轮廓 } & 类型 & \multicolumn{4}{c}{ 次数 } \\
\cline { 3 - 6 } & & 5 & 10 & 20 & 25 \\
\hline \multirow{2}{*}{$\mathrm{A}$} & 截面单元/个 & 126 & 479 & 1893 & 2958 \\
& 截面点/个 & 210 & 799 & 3155 & 4930 \\
& 边界轮廓线段/个 & 68 & 132 & 264 & 332 \\
\hline \multirow{2}{*}{ B } & 截面单元/个 & 344 & 1500 & 5846 & 9126 \\
& 截面点/个 & 573 & 2500 & 7743 & 9743 \\
& 边界轮廓线段/个 & 124 & 255 & 506 & 636 \\
\hline \multirow{2}{*}{$\mathrm{C}$} & 截面单元/个 & 129 & 520 & 2079 & 3237 \\
& 截面点/个 & 215 & 867 & 3465 & 5395 \\
& 边界轮廓线段/个 & 124 & 138 & 282 & 350 \\
\hline
\end{tabular}

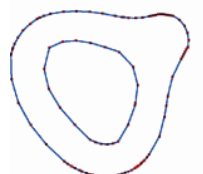

(a)

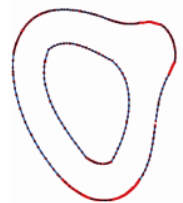

(c)

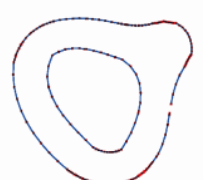

(b)

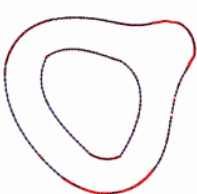

(d)
图 21 不同细分次数的截面边界轮廓线段

HNURBS 体离散的六面体单元个数 $n$, 以某一 切片截面为例, 算法时间复杂度分析如下: 在最坏 情况下, 需要遍历 $n$ 个六面体单元与切片截面求交, 由于对截面点排序及剔除 “伪边界轮廓线段” 的算 法时间复杂度均为 $O(1)$, 而哈希表构建和排序的算 法时间复杂度为 $O(n)$, 因此整体切片算法时间复杂 度为 $O(n)$ 。支撑点求解过程中, 由于截面点数量和 六面体单元个数 $n$ 呈线性关系，因此支撑点求解的 算法时间复杂度为 $O(n)$ 。表 4 所示为图 20c 位置三 个截面依次在细分 5 次、10 次、20 次、25 次的情 况下, 离散的六面体单元个数, 切片及支撑点求解 过程中所消耗的时间, 机器配置为 Intel Xeon 3.40 $\mathrm{GHz}$ ，运行内存为 $16 \mathrm{~GB}$ 。

表 4 不同细分次数切片与支撑点求解时间

\begin{tabular}{cccccc}
\hline \multirow{2}{*}{$\begin{array}{c}\text { 边界 } \\
\text { 轮廓 }\end{array}$} & 类型 & \multicolumn{4}{c}{ 次数 } \\
\cline { 3 - 6 } & & 5 & 10 & 20 & 25 \\
\hline & 六面体单元/个 & 3375 & 27000 & 216 & 421875 \\
& 切片过程/ms & 57 & 394 & 2943 & 6101 \\
\multirow{2}{*}{$\mathrm{A}$} & 支撑求解过程/ms & 3 & 10 & 42 & 64 \\
& 切片过程/ms & 110 & 803 & 4384 & 8482 \\
\multirow{2}{*}{ B } & 支撑求解过程/ms & 25 & 105 & 426 & 644 \\
& 切片过程/ms & 58 & 465 & 3141 & 5551 \\
C & 支撑求解过程/ms & 3 & 12 & 48 & 85 \\
\hline
\end{tabular}

图 22 所示为针对 Block 模型相邻两截面求取 截面点的过程示意图。图 22a、22b、22c 为沿箭头 切片生长方向三个依次升高的相邻截面边界轮廓 $\mathrm{A} 、 \mathrm{~B} 、 \mathrm{C}$ 。图 $22 \mathrm{~d}$ 为 $\mathrm{B}$ 轮廓在 $\mathrm{A}$ 轮廓的基础之上, 寻找 $\mathrm{B}$ 轮廓完全位于 $\mathrm{A}$ 轮廓之外的区域作为支撑 区域, 在支撑区域加上支撑点, 如图中点所示。图 $22 \mathrm{e}$ 为 $\mathrm{C}$ 轮廓在 $\mathrm{B}$ 轮廓基础之上得到的支持区域和 支撑点。 


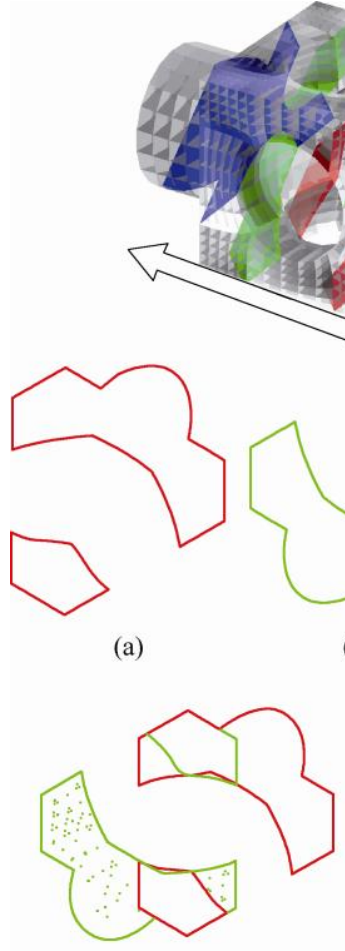

(d)

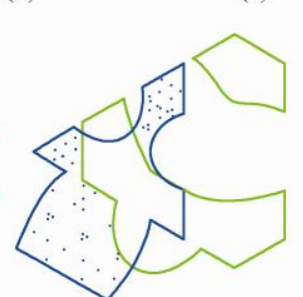

(e)
图 22 相邻两层之间的支撑点求解过程

图 23 所示为四个 HNURBS 体分别沿 $x, y, z$, 任意方向的切片结果和求解支撑点实例图。其中箭 头表示切片生长方向, 模型上彩色点表示需要添加 支撑点的位置。

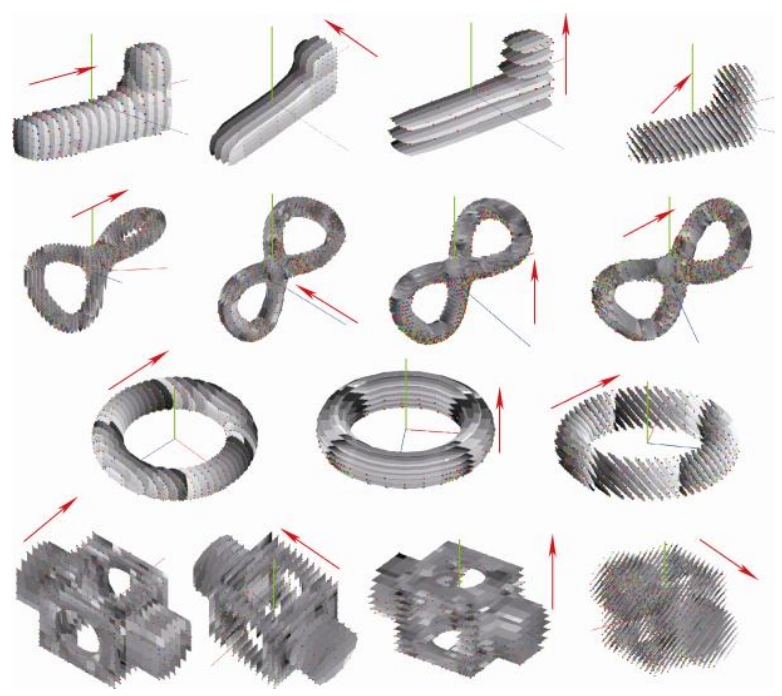

图 23 HNURBS 体的切片及支撑点求解

\section{6 结论}

本文首先构建复杂的多片 NURBS 体参数化模 型, 通过对模型控制点赋予材料信息, 形成复杂的 多片 HNURBS 体。结合 NURBS 体参数化模型的细 分方法, 将 HNURBS 体划分为六面体单元模型, 利
用平面求交和轮廓提取得到一系列切片的轮廓区域 数据, 通过轮廓区域数据的拟合得到三维打印切平 面, 最后根据相邻两层截面的布尔运算求解得出支 撑点。

不同于现有的切片算法，本文为非均质的、多 片的、拓扑复杂的体参数化模型的三维打印技术提 供了算法支持。由于体参数模型在表达非均质材料 方面具有极大的优势，因此本文方法在非均质产品 三维打印方面将具有很大的发展前景。后期工作将 致力于支撑点的优化, 以及三维打印路径规划, 且 形成可供三维打印机使用的含有材料信息的 G-CODE 代码。

\section{参 考 文 献}

[1] NGO T D, KASHANI A, IMBALZANO G, et al. Additive manufacturing (3D printing): A review of materials, methods, applications and challenges $[\mathrm{J}]$. Composites Part B Engineering, 2018， 143: 172-179.

[2] LIVESU M, ELLERO S, MARTíNEZ J, et al. From 3D models to 3D prints: An overview of the processing pipeline[J]. Computer Graphics Forum, 2017, 36(2): 537-564.

[3] NORMAN J, MADURAWE R D, MOORE C M, et al. A new chapter in pharmaceutical manufacturing : 3D-printed drug products[J]. Advanced Drug Delivery Reviews, 2016, 108: 39-50.

[4] YIN L, JONATHAN D, TIAN X, et al. Design and characterization of radar absorbing structure based on gradient-refractive-index metamaterials $[\mathrm{J}]$. Composites Part B Engineering, 2018， 132: 178-187.

[5] TURCO E, GOLASZEWSKI M, GIORGIO I, et al. Pantographic lattices with non-orthogonal fibres : Experiments and their numerical simulations[J]. Composites Part B Engineering, 2017, 118: 1-14.

[6] WU P, WANG J, WANG X. A critical review of the use of 3 -D printing in the construction industry[J]. Automation in Construction, 2016, 68: 21-31.

[7] KIEBACK B, NEUBRAND A, RIEDEL H. Processing techniques for functionally graded materials $[\mathrm{J}]$. Materials Science \& Engineering A, 2003， 362(1): 81-105.

[8] JÜTTLER B, SIMEON B. Isogeometric analysis and applications 2014[M]. London: Springer International Publishing, 2015.

[9] JAKSON T, LIU H, PATRIKALAKIS N, et al. Modeling and designing functionally graded material components for fabrication with local composition control[J]. Materials \& Design, 1999, 20(2-3): 63-75.

[10] BISWAS A, SHAPIRO V, TSUKANOV I. Heterogeneous 
material modeling with distance fields[J]. Computer Aided Geometric Design, 2004, 21(3): 215-242

[11] WANG S, CHEN N, CHEN C S, et al. Finite element-based approach to modeling heterogeneous objects[J]. Finite Elements in Analysis \& Design, 2009, 45(8): 592-596.

[12] WU X, LIU W, WANG M Y. A CAD modeling system for heterogeneous object[J]. Advances in Engineering Software, 2008, 39(5): 444-453.

[13] CHOI S, CHEUNG H. A topological hierarchy-based approach to layered manufacturing of functionally graded multi-material objects[J]. Computers in Industry, 2009, 60(5): 349-363.

[14] LIU H, MAEKAWA T, PATRIKALAKIS $\mathrm{N}$, et al. Methods for feature-based design of heterogeneous solids[J]. Computer-Aided Design, 2004, 36(12) : 1141-1159.

[15] COHEN E, MARTIN T, KIRBY R, et al. Analysis-aware modeling : Understanding quality considerations in modeling for isogeometric analysis[J]. Computer Methods in Applied Mechanics and Engineering, 2010, 199(5-8): 334-356.

[16] MARTIN T , COHEN E , KIRBY R. Volumetric parameterization and trivariate $\mathrm{B}$-spline fitting using harmonic functions[J]. Computer Aided Geometric Design, 2009, 26(6): 648-664.

[17] AIGNER M, HEINRICH C, JÛ TTLER B, et al. Swept volume parameterization for isogeometric analysis[C]// Ima International Conference on Mathematics of Surfaces Xiii. Springer-Verlag, 2009: 19-44.

[18] WANG X, QIAN X. An optimization approach for constructing trivariate B-spline solids[J]. Computer-Aided Design, 2014, 46(1): 179-191.

[19] XU G, MOURRAIN B, GALLIGO A. High-quality construction of analysis-suitable trivariate NURBS solids by reparameterization methods $[\mathrm{J}]$. Computational Mechanics, 2014, 54(5): 1303-1313.

[20] XU G, KWOK T, WANG C C. Isogeometric computation reuse method for complex objects with topology-consistent volumetric parameterization [J]. Computer-Aided Design, 2017, 91: 1-13.

[21] XIAO S, KANG H, FU X M, et al. Computing IGA-suitable planar parameterizations by poly square-enhanced domain partition[J]. Computer Aided Geometric Design, 2018，62: 29-43.

[22] SUN S, CHIANG H, LEE M. Adaptive direct slicing of a commercial CAD model for use in rapid prototyping $[\mathrm{J}]$. International Journal of Advanced Manufacturing
Technology, 2007, 34(7-8): 689-701.

[23] MINETTO R, VOLPATO N, STOLFI J, et al. An optimal algorithm for 3D triangle mesh slicing $[\mathrm{J}]$. Computer-Aided Design, 2017, 92: 1-10.

[24] DING D, PAN Z, CUIURI D, et al. Automatic multi-direction slicing algorithms for wire based additive manufacturing $[\mathrm{J}]$. Robotics and Computer-Integrated Manufacturing, 2016, 37: 139-150.

[25] SONG Y, YANG Z, LIU Y, et al. Function representation based slicer for 3D printing $[\mathrm{J}]$. Computer Aided Geometric Design, 2018，62: 276-293

[26] MA W, BUT W, HE P. NURBS-based adaptive slicing for efficient rapid prototyping[J]. Computer-Aided Design, 2004, 36(13): 1309-1325.

[27] ALEXANDER P, ALLEN S, DUTTA D. Part orientation and build cost determination in layered manufacturing $[\mathrm{J}]$. Computer Aided Design, 1998, 30(5): 343-356.

[28] CHEN Y, LI K, QIAN X. Direct geometry processing for telefabrication[J]. Journal of Computing \& Information Science in Engineering, 2013, 13(4): 411-424.

[29] JÉRÉMIE D, HERGEL J, LEFEBVRE S. Bridging the gap: Automated steady scaffoldings for 3D printing[J]. ACM Transactions on Graphics, 2014, 33(4): $1-10$

[30] HUANG X, YE C, MO J, et al. Slice data based support generation algorithm for fused deposition modeling $[\mathrm{J}]$. Tsinghua Science \& Technology, 2009, 14(S1): 223-228.

[31] 陈岩, 王士玮, 杨周旺, 等. FDM 三维打印的支撑结构 的设计算法 $[\mathrm{J}]$. 中国科学：信息科学, 2015, 45(2): 259-269.

CHEN Yan, WANG Shiwei, YANG Zhouwang, et al. Construction of support structure for FDM 3D printers[J]. Scientia Sinica Informationis, 2015，45(2): 259-269.

[32] CHEN L, XU G, WANG S Y, et al. Constructing IGA-suitable volumetric parameterization based on directed graph simplification of $\ell_{1}$ polycube structure from complex shapes[J]. Computer Methods in Applied Mechanics and Engineering, 2019， 351: 422-440.

[33] 陈龙, 张军洋, 李郝林, 等. 面向材料反求的非均质体 参数化模型构建 $[\mathrm{J}]$. 机械工程学报, 2014, 50(23): 156-164.

CHEN Long, ZHANG Junyang, LI Haolin, et al. Reverse-material-oriented heterogeneous volume parameterization model construction[J]. Journal of Mechanical Engineering, 2014, 50(23): 156-164.

作者简介: 陈龙(通信作者), 男, 1978 年出生, 博士, 副教授, 硕士研 究生导师。主要研究方向为产品计算设计, 机器视觉。

E-mail: cl@usst.edu.cn 